\title{
Evaluation of Cart Fill Drug Distribution System for In-patients at a South Indian Tertiary Care Teaching Hospital
}

\author{
Dilna Raveendran, Adepu Ramesh*, Justin Kurian \\ Department of Pharmacy Practice, JSS College of Pharmacy, JSS University, Mysuru - 570 015, INDIA.
}

\begin{abstract}
Accurate and safe distribution of medications to the patients is an integral responsibility of the hospital pharmacy. Various international studies have shown the efficiency of cart fill method of drug distribution in ensuring medication accuracy. Objective: To assess the efficacy of cart fill drug distribution system at a South Indian Tertiary care teaching hospital. Methodology: A suitable data collection form for cart fill drug distribution system was designed. Standard operating procedure (SOP) was prepared to implement the method of cart fill drug distribution. As per the SOP, the duty pharmacist verified the e-prescriptions for transcribing errors. The research pharmacist verified the prescription for any potential drug related problems, and cross verified the filled medications with prescription for accuracy and then filled medicines were handed over to the patients with instructions for safe use. A separate feed- back questionnaire was applied to collect the clinicians and patients satisfaction over the cart fill drug distribution practice. Results: During the study period, about 5000 prescriptions were reviewed and identified $502(10.4 \%)$ errors and corrected. Majority errors found were dispensing errors $(64.14 \%)$, and transcribing errors $(17.52 \%)$. At the end of the study, significant reduction $(p<0.01)$ in errors was observed. Number of drug returns was also found decreased. Average time taken for handing over the medicines to the patient was calculated as less than 45minutes. Clinicians and Patients have expressed satisfaction over this system of drug distribution. Conclusion: The present cart fill drug distribution system has ensured accuracy in medication supply within the scheduled time and decreased the number of drug returns to the pharmacy and also increased the patient's satisfaction.
\end{abstract}

Key words: Cart-fill drug distribution, Transcribing errors, Dispensing errors, E-Prescription, and Drug returns.

\section{INTRODUCTION}

Drug distribution is an important professional responsibility of the hospital pharmacists. The unit dose distribution of drugs is a pharmacy coordinated method which was developed and practiced since 1960's for distributing medications in an organized way. Most of the European countries follow the unit dose drug distribution for dispensing medicines to their patients and currently Germany is also trying to implement unit dose drug distribution in the hospitals. ${ }^{1}$ In Canada, $22 \%$ of the hospitals use decentralised unit dose drug distribution and the trend currently increasing in other hospitals also. ${ }^{2}$ Even in Middle East countries, the drug distribution practice in the hospitals is more towards decentralised. In Saudi Arabia,

DOI: 10.5530/ijopp.9.3.5

$21 \%$ of the hospitals use decentralized method of drug distribution and more importantly use the bar code system that ensures the accuracy of medication and distribution to the right patients. ${ }^{3}$ In India, majority hospitals use traditional drug distribution practices such as individual prescription order method and centralized unit dose drug distribution. These practices incur excess of inventory, increased manpower, huge financial burden on the hospital and decreased patient safety.

In unit dose distribution system, medications are dispensed in unit doses, where each dose of medication is separately packed and labelled with the drug name, strength, lot number and expiration date in a ready
Address for correspondence:

Dr. Ramesh Adepu, Professor,

Department of Pharmacy Practice,

JSS College of Pharmacy, JSS University,

Sri Shivarathreeshwara Nagar, Mysuru- 570 015, INDIA.

Mobile: +9199455 20215

E-mail: adepu63@gmail.com

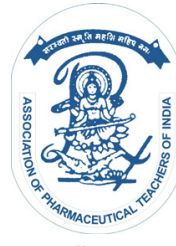

www.ijopp.org 
to administer form. In this system, the pharmacist becomes more actively involved in analysing the prescriptions for potential errors that creep in while transcribing or while dispensing. Despite the precautions taken by the pharmacists, errors may likely take place that affects the patients' desired therapeutic outcomes. So the quest was on to come out with a most suitable method that ensures appropriateness in the dispensed medicines.

Among the available new methods of drug distribution, Cart fill drug distribution is a new innovative method of drug distribution practices that improvised the efficiency of drug distribution that has reduced the duration between drug order and receipt time and also drug returns to the pharmacy. ${ }^{4}$ Cart fill drug distribution system is a decentralized drug distribution practice in which a portable cart is used. The cart contains separate drawers for stocking medicines for separate patients. Medications are prepared by the pharmacists in the central hospital pharmacy and supplied round the clock. ${ }^{5}$ the cart fill method of drug distribution allows the pharmacy to dispense the number of doses needed by individual patient during his/her 24 hours of hospital stay. This system allows the multiple checking of the medication before administration to the patient.

JSS Hospital is a South Indian Tertiary care teaching hospital with 1800 bed multi specialities with $85 \%$ bed occupancy rate. In order to provide efficacious distribution of medicines to the in-patients, the cart fill method of drug distribution was introduced. Thus, the present study was focused to evaluate the efficacy of cart fill drug distribution system in terms of incidence of prescription errors, dispensing time and patient satisfaction at JSS Hospital.

\section{METHODOLOGY}

The study was conducted as a pilot study in the satellite pharmacies of private wards and critical care unit of JSS Hospital, Mysuru. JSS Hospital is 1800 bed multispeciality tertiary care teaching hospital and provides healthcare facilities to people in and around Mysuru district. The study was a prospective interventional study conducted over a period of nine months during July 2015 to March 2016. For conducting the study, a standard operating procedure (SOP) was prepared.

As per SOP, a team of 2 technicians from Pharmacy, 1 duty pharmacist and 1 research pharmacist were made as a group for each satellite pharmacy. On receipt of online prescription in the pharmacy, the duty pharmacist verifies the accuracy of the prescription. Upon approval, the pharmacy technician fills the prescription.
The research pharmacist further verifies the drugs dispensed against the prescription before they are sent to the patient's bedside through cart. All the drugs are placed in a separate cover labelled with patient details and the copy of the prescription and in case of solid dosage form drugs are placed in a cover that contain patient details and frequency of administration etc. The research pharmacist then place the medication into medication cart containing drawers for each patient. Every forty five minutes the pharmacy technician is going to the wards and the research pharmacist also going along with them and distributes the medication to the respective nursing staff. Again the nursing staff cross check the medications. The research pharmacist cross verify the medications with patient's medication chart before handing over the medications to the patient. The research pharmacist also brief the patient or patient's representative regarding the safe use of their medicines. The efficiency of the cart fill method of drug distribution is assessed by parameters such as,

1. Number of medication errors encountered and corrected

2. Number of drug returns

3. Time efficiency in supplying the medicines to patients and

4. Clinicians and Patients feedback

\section{RESULTS}

A total of 5000 prescriptions were reviewed during the study period. Among them, 502 errors (10.4\%) were identified. Of which, 322 were dispensing errors (64.14\%), 88 were transcribing errors $(17.52 \%), 85$ were prescription errors $(16.93 \%)$, and 7 were administration errors $(1.39 \%)$. All the errors were corrected. The details of the same are presented in Figure 1.

Among 322 dispensing errors, 114(35.4\%) were observed as missing medication, $64(19.87 \%)$ were observed as wrong dispensing, $69(21.4 \%)$ were found as over dispensing, 75(23.29\%) were found as under dispensing. The details of the different types of dispensing errors are presented in Figure 2.

\section{Drug Returns}

During the study period, 84(1.7\%) drugs were returned to the pharmacy due to various reasons. Among them, 31 were due to change in the drug $(36.9 \%), 36$ were due to change in the dose $(42.85 \%), 15$ were due to change in the formulation $(17.85 \%)$, and 2 were due to change in prescription $(2.38 \%)$. The details of drug returns are presented in Figure 3.

Indian Journal of Pharmacy Practice, Vol 9, Issue 3, Jul-Sep, 2016 


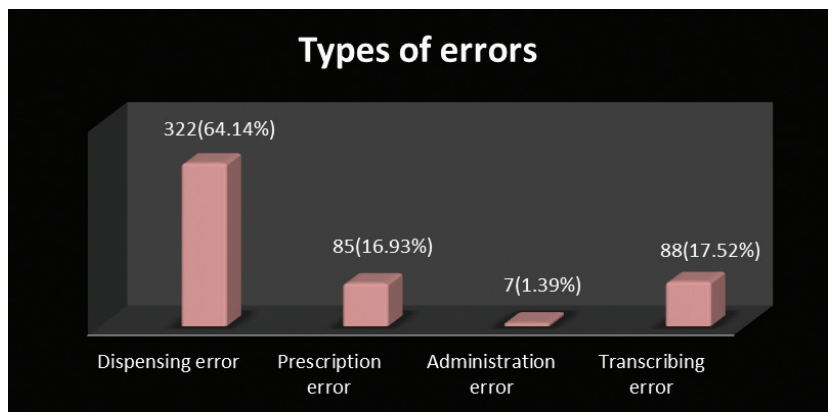

Figure 1: Types of Errors observed during the drug distribution.

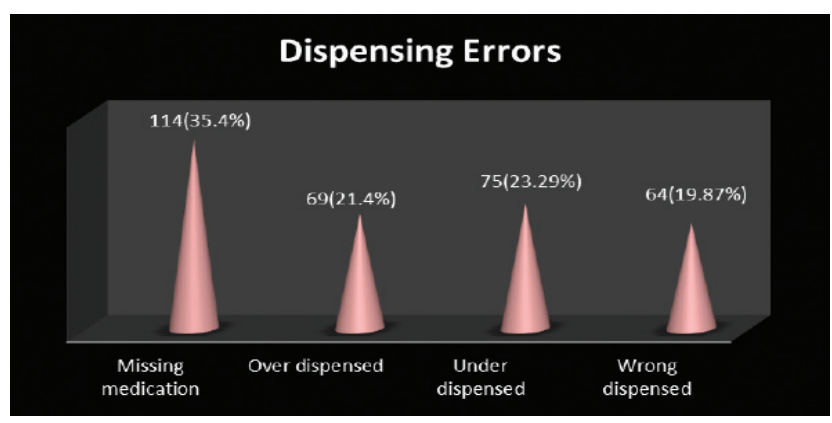

Figure 2: Types of dispensig errors.

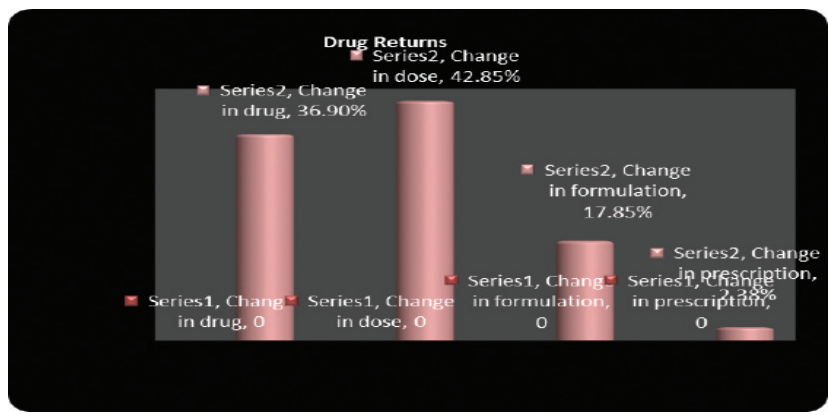

Figure 3: Reasons Drug Returns.

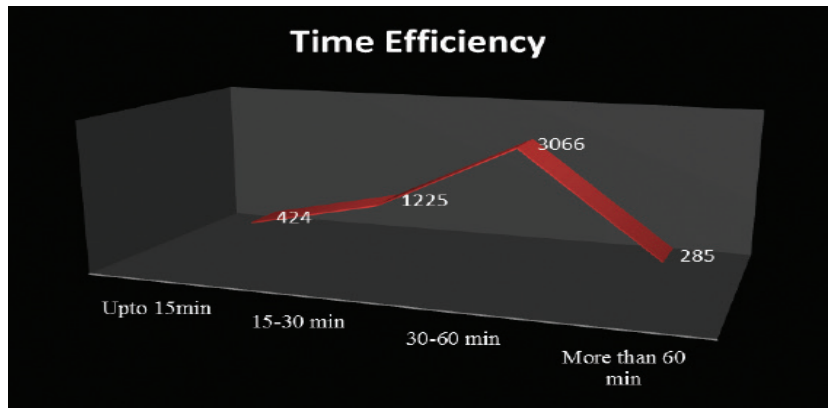

Figure 4: Time duration in distributing the medicines.

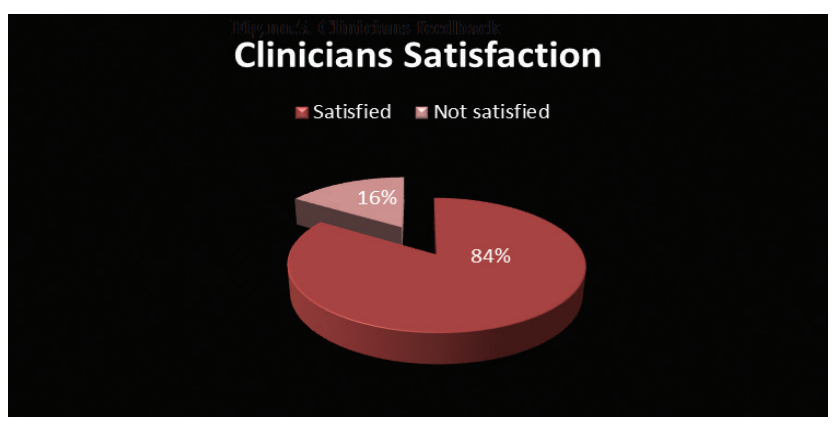

Figure 5: Clinians Feedback.

\section{Time Efficiency}

During the study period, a total of 5000 prescriptions were reviewed. The average time spent to dispense these prescriptions was analysed. Among them 424 prescriptions were dispensed, within 15 minutes (8.48\%),1225 prescriptions were dispensed within 15-30minutes $(24.5 \%), 3066$ prescriptions were dispensed within 30-60minutes (61.32\%), and 285 prescriptions were dispensed taking more than an hour $(5.7 \%)$. The details are presented in Figure 4.

\section{Patient Feedback}

Perception and opinion of patients towards the cart fill drug distribution system was analysed. During the study, a total of 800 patients from the private medical wards were interviewed to assess their satisfaction with this service during the study period. Among them, 585 patients had expressed that they were very much satisfied $(73.12 \%)$ and 204 patients had expressed $(25.5 \%)$ satisfaction over the service. Only 11 patients have expressed that they were not satisfied $(1.37 \%)$ with the service.

\section{Clinicians Feedback}

Clinician satisfaction feedback questionnaires were circulated among 25 Clinicians who visit these wards where the cart fill drug distribution system is practiced.

Perception and opinion of Clinicians towards cart fill drug distribution system have been analysed through the answers provided. Out of 25 clinicians, 21 clinicians have expressed that they were satisfied with this system of drug distribution. The details are presented in Figure 4.

\section{DISCUSSION}

On a pilot basis, cart fill drug distribution system was introduced only in the private wards and critical care unit of JSS Hospital for ensuring the quick supply of medicines to the patients.

During the study period, $10.4 \%$ errors were identified and corrected. The most common errors found were dispensing errors $(64.14 \%)$, transcribing errors $(17.52 \%)$, prescription errors $(16.93 \%)$ and administration errors (1.39\%). In a study conducted by Lisby $\mathrm{M}$ et al, had detected a total of 1065 errors in 2467 prescriptions. Among them 56\% was transcription errors, $4 \%$ were dispensing errors and $14 \%$ were administration errors. ${ }^{6}$ In our study, more of dispensing errors were observed compared to the transcribing errors. Reasons for dispensing errors was observed as high prescription volume, heavy work load, similar or confusing drug names (sound alike and look alike drugs). However due to the research pharmacist intervention in verification filled medication order, the errors frequency was decreased. 
In another study conducted by Cina JL et al, in US in 2006 observed that high incidence rate of dispensing errors is mainly due to sound alike and look alike medicines and the decreased rate of dispensing errors is mainly due to the cross verification of filled medication with prescription. ${ }^{7}$ Similar findings are observed in our study also. Dispensing errors can be minimized by verifying patient identity while entering the prescription into the computer, check the accuracy of prescription before dispensing, store the sound alike and look alike medicine different shelf with proper labels, recheck the filled medication order before handing the medication to the patients.

Transcribing error is a specific type of medication error and occurs while entering the medicine names, doses or frequency wrongly in the computer. A total of $17.52 \%$ transcribing errors were identified during the study. Most of the transcribing errors were an omission error which means the prescribed drug did not reach the patient, wrong dose, alternative drug selection, requesting more quantity of drugs than prescribed, unauthorized medication etc. A study conducted by Fanak Fahimi et al in a tertiary care teaching hospital in Iran showed that, the rate of transcribing errors happened while using the manual prescribing system. A total of 287 medication charts were reviewed. About 289 errors were identified. Omission errors were found more in transcribing errors. ${ }^{8}$

In our study, out of the 5000 prescriptions, only few were resulted into transcribing error. These were due to the electronic prescription system. More number of transcribing errors was observed during the initial stages of the study. Training given to the nursing staff by the research pharmacist resulted in reduction in transcribing error in the later stages of the study.

Prescribing medicine to patient is an integral part of medical care. Of the 5000 prescriptions reviewed, $16.93 \%$ prescription errors were identified and corrected. Most common errors identified were drug duplication, prescribing the drugs that have increased chances of interaction, wrong dose, wrong route of administration, wrong frequency of administration etc. In a study conducted by Lt Col Prafull Mohan et al, among the randomly collected 1000 prescriptions, the researchers had observed that 650 prescriptions $(65 \%)$ were found to possess a total of 1012 errors. Type B errors were observed in $22.4 \%$ prescriptions, type $\mathrm{C}$ errors were found in $9.7 \%$ prescriptions and types $\mathrm{D}$ were found in $69.1 \%$ prescriptions. This study necessitates the need for a regular structured prescription audits to minimize the prescription errors. ${ }^{9}$

Prescription errors can be minimized by keeping the patient medication list up-to- date in a medical record. This system helps in avoiding medication duplication, potential drug interactions, prescribing contraindicated medications, wrong route, wrong frequency of administration. The electronic prescription system can be integrated with patient details including his/her physiological parameters, known allergies, real time information regarding availability of medicines. This integrated system will help to prevent the prescription errors.

Medication administration is an important part of delivering safe patient care. Administration errors include the following; wrong drug, wrong route, wrong dose, wrong patient, wrong timing of drug administration, a contraindicated drug for that patient, wrong site, wrong drug form, wrong infusion rate, expired medication date or prescription. About $1.39 \%$ errors were found as administration errors in the study. Low incidence of administration errors are mainly due to significant improvement in the nursing care.

K W Ridge et al in their study reported a much higher error frequency. They conducted a cohort observational study for a period of 4 months. They had observed 74 drug rounds in which 115 errors were observed during 3312 drug administrations. The overall error rate was found to be $3.5 \%$ (95\% confidence interval $2.9 \%$ to $4.1 \%$ ). Errors owing to omissions were accounted for $68 \%$ (78) because the drug had neither been supplied nor located with patient or the prescription was present. Wrong doses accounted for 15\% (17) of the errors. Four of them were greater than the prescribed dose. The dose was given within two hours of the time indicated by the prescriber in $98.2 \%$ of cases. ${ }^{10}$

After dispensing the medicine there is an increased chance of returning the medication back to the pharmacy. The relevant reasons included, wrong medication, change of medication, sound alike look alike drugs, improperly stored drugs, dispensing error, subsidence of symptoms and death of the patient. In the present study drug returns were found as only $1.7 \%$. Change in dose, change in drug, change in formulation and change in prescription, were the main reasons for drug returns. However the drug returns percentage was drastically reduced due to cross verification of prescriptions by the research pharmacist. A similar type of finding was observed in a study conducted by Korby Lathrop et al. After the implementation of thrice daily cart fill in the medical center pharmacy resulted in $42.9 \%$ decrease in the number of medications returned to central pharmacy. ${ }^{11}$

Implementation of cart fill drug distribution system resulted in timely delivery of medication to the patient. Most of the medications were dispensed to the patient within 30-60 minutes. Few prescriptions were dispensed by taking more than 60 minutes. Reasons for delay in filling the prescriptions were due to some of the drugs

Indian Journal of Pharmacy Practice, Vol 9, Issue 3, Jul-Sep, 2016 
were out of stock in the pharmacy and the technician had to collect the drugs from the central pharmacy.

\section{Patient Feedback}

Client satisfaction is the key element in any services. In pharmaceutical care service patient satisfaction plays a prime role. When this new service was introduced, patient satisfaction was considered as one of the measures to assess efficiency of the service. $73.15 \%$ patients were very much satisfied over the system. $25.5 \%$ were expressed that were satisfied and 1.37\% were not satisfied with this system. Most of the patients get all the medication within the time. Only few of the patients are not counselled due to lack of time and emergency requirement of the medicines. Remaining all the patients was educated about the drugs, disease, and life style modification. In this study, patients were satisfied with this system due to quick availability of prescribed medicines, and receiving the necessary information about safe use of their medications.

\section{Clinicians Feedback}

Clinician's satisfaction is an important aspect of this study. Clinicians were satisfied with the education support provided to the patient, and found helpful in improving patient medication adherence, behaviour, and clinical outcomes. About $86 \%$ of the clinicians have expressed their satisfaction over the system. The suggestions given by the clinicians to improve the system includes to educate discharging patients regarding the safe use of medication, expand this system across the hospital and provide education to the nursing staff related to medication.

In a study conducted by Elise Rochais et al, on nursing staff's perception and satisfaction over medication carts dispensing in the point of care units. Total of 195 nurses completed the questionnaire. Among them, 64\% of nurses have agreed that the service had reduced the risk of interruption \& $45 \%$ of the nurses have agreed that the service has reduced risk of their colleague's interruption. ${ }^{12}$

\section{CONCLUSION}

The findings of the study conclude that, the newly introduced Cart fill drug distribution system has decreased the transcribing, dispensing and administration errors and also the drug returns to the pharmacy. Most of the inpatients and their caretakers have expressed satisfaction over the drug distribution. Clinicians have also expressed their satisfaction over this cart fill method of drug distribution system.

\section{ACKNOWLEDGEMENTS}

The authors are thankful to Principal, JSS College of Pharmacy, Mysuru, JSS University, Director and Medical Superintend, JSS Hospital, In-charge, Hospital Pharmacy department for providing the needful help to complete this research work.

\section{CONFLICT OF INTEREST}

None.

\section{ABBREVIATION USED}

Nil

\section{REFERENCES}

1. Beard R. Drug dispensing system in the UK time for rethink. Hospital Pharm Europe. 2010;(49). PMid:21040741.

2. Clark C. UDSS were once missed as an impossible dream but electronic prescribing and closed loop medication administration system make this the logical way to deliver the medication safely and cost effectively. Hospital Pharm Europe. 2014;(7). PMid:25004594.

3. Alsultan MS, Khurshid F, Mayet AY. Hospital pharmacy practice in Saudi Arabia:Dispensing and administration in the Riyadh region. Saudi Pharmaceutical J. 2012;20(4):307-15. http://dx.doi.org/10.1016/j. jsps.2012.05.003 ; PMid:23960805 PMCid:PMC3745065.

4. Dandiya PC, Mukul M. Hospital \& Clinical pharmacy .3rd ed. Delhi: MK Jain Publishers. 2002:24-32.

5. Bajaj A, Tipnis HP Hospital pharmacy. Ist ed. Maharashtra: Career publication; 2007:89-98.

6. Lisby M, Nielsen LP, Mainz J. Errors in the medication process: frequency, type, and potential clinical consequences. Int J Qual Health Care. 2005;17(1):15-22. http://dx.doi.org/10.1093/intqhc/mzi015 ; PMid:15668306.

7. Cina JL, Gandhi TK, Churchill W et al. How many hospital pharmacy medication dispensing errors go undetected?. Comm J Qual Patient Saf. 2006;32(2):73-80.

8. Fahimi F, Nassari MA, Abrishami R, et al. Arch Iranian Med. Transcription Errors Observed in a Teaching Hospital. 2009;12(2):173-5.

9. Mohan P, Sharma AK, Panwar SS. Identification and quantification of prescription errors. Medical J Armed Force India. 2014;70(2):149-53. http:// dx.doi.org/10.1016/j.mjafi.2014.01.002 ; PMid:24843204 PMCid:PMC4017179.

10. Ridge KW, Jenkins DB, Noycee PR et al. Medication errors during hospital rounds. Int J Qual Health Care. 1995;1;4(1):240-3.

11. Lathrop K, Lund J, Ludwig B et al. Design implementation and evaluation of thrice daily cart fill process. Am J Health-Syst Pharm. 2014;71(13):1112-9. http://dx.doi.org/10.2146/ajhp130411; PMid:24939501.

12. Rochais E, Atkino BJF. Nursing perception of the impact of medication carts on patient safety and ergonomics in a teaching health care center. J Pharm Pract. 2013;26(2):131-7. http://dx.doi.org/10.1177/0897190012451908; PMid:22797833. 